\title{
REVIEW
}

\section{Endoplasmic reticulum quality control and congenital pathology}

\author{
Marek Michalak \\ Department of Biochemistry, University of Alberta, Edmonton, Alberta, Canada
}

Received $6^{\text {th }}$ September 2005.

Published online $12^{\text {th }}$ October 2004.

\begin{abstract}
Summary
Quality control of the endoplasmic reticulum plays a critical role in protein folding, modification and modification of a secretory pathway. As endoplasmic reticulum chaperones, calreticulin and calnexin have similar substrate specificity and share several common features. Yet, surprisingly, mice bearing a disruption in the calreticulin gene die from a lesion in cardiac development and develop significant metabolic problems whereas calnexin-deficient mice are born alive with, yet not understood, neurological problems. Studies with calreticulin and calnexin gene knockout mice and calreticulin- and calnexindeficient cell lines indicate that calnexin is unable to compensate for the loss of calreticulin and conversely, calreticulin cannot compensate for the loss of calnexin. Calreticulin or calnexin deficiency or reduction in the level of ERp57 protein (ERp57 heterozygote mice) leads to development of metabolic disorders as documented by sever changes serum lipids and carbohydrates composition in these animals. These observations indicate that calreticulin, calnexin and ERp57, in addition of being involved in maturation of glycoproteins in the endoplasmic reticulum, perform other distinct functions including affecting energy metabolism.
\end{abstract}

Keywords: endoplasmic reticulum - calreticulin - calnexin - chaperones - lipid metabolism

\section{INTRODUCTION}

Many human diseases are caused by mutations altering the folding pathway and final conformation of a protein. Many conformational diseases are caused by mutations in secretory proteins and leading to metabolic dysfunctions i.e. diabetes, to

Marek Michalak, Department of Biochemistry, University of Alberta, 3-56 Medical Sciences Building, Edmotnon, Alberta, Canada T6G 2H7

몽.marek.michalak@ualberta.ca

용 $+780-492-2256$

㚗 $+780-492-008$ development and neurological diseases such as Alzheimer's. The endoplasmic reticulum (ER) is a processing place for the maturation, folding, transport and storage of proteins, and is also the most prominent intracellular $\mathrm{Ca}^{2+}$ store (Baumann and Walz 2001). The ER contains many proteins which carry out these diverse functions (Baumann and Walz 2001, Corbett and Michalak 2000, Meldolesi and Pozzan 1998, Trombetta and Parodi 2003). Calreticulin is an ER luminal $\mathrm{Ca}^{2+}$ binding chaperone involved in regulation of intracellular $\mathrm{Ca}^{2+}$ homeostasis and ER $\mathrm{Ca}^{2+}$ capacity (Arnaudeau et al. 2002, Nakamura et al. 2001). This is important because changes in the $\mathrm{ER} \mathrm{Ca}^{2+}$ storage capacity affect its chaperone function and influence the quality control of the secretory pathway (Corbett et al. 2000). Calnexin is a type I integral 
membrane chaperone of the ER (Wada et al. 1991). The protein has a high degree of amino acid sequence and structural similarity (identity) to calreticulin (Bergeron et al. 1994, Michalak et al. 1999, Michalak et al. 2002). Calreticulin and calnexin, together with ERp57 (a PDI-like protein with thoredoxin domains which is also resident in the ER) constitute the calreticulin/calnexin cycle that is responsible for the folding and quality control of newly-synthesized glycoproteins (Trombetta and Parodi 2003). As ER chaperones, calreticulin and calnexin have similar substrate specificity and share several common features (Trombetta and Parodi 2003). Surprisingly, mice bearing a disruption in the calreticulin gene die from a lesion in cardiac development (Mesaeli et al. 1999), develop significant metabolic problems (Guo et al. 2002) whereas calnexin-deficient mice

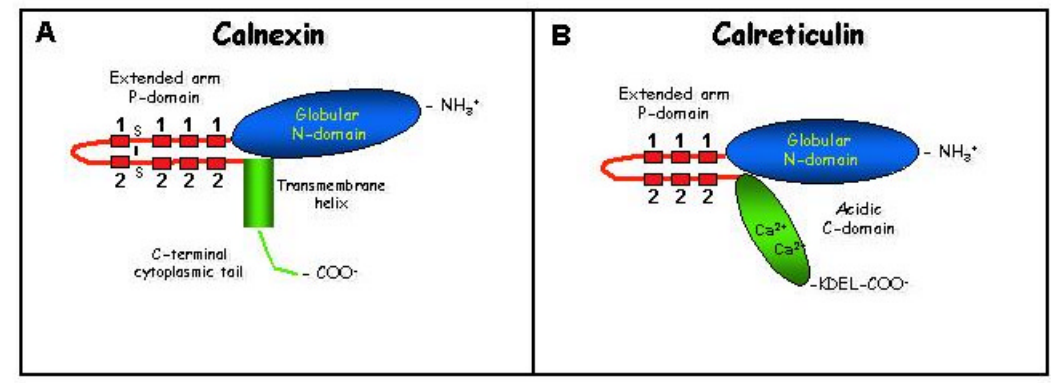

Fig. 1. A model of calnexin (A) and calreticulin (B) domains. Calreticulin contains a globular N-domain (blue) and central, proline-rich P-domain (red) which forms a characteristic loop. C-terminal C-domain (green) contains a large number of negatively charged amino acids and it is involved in high capacity $\mathrm{Ca}^{2+}$ storage. Similar domain arrangement is found in calnexin with exception that calnexin is an integral membrane protein with a C-terminal trasmembrane helix and cytoplasmic tail.

are born alive with, yet not understood, neurological problem (Denzel et al. 2002). Studies with calreticulin and calnexin gene knockout mice and calreticulin- and calnexin-deficient cell lines indicate that calnexin is unable to compensate for the loss of calreticulin (Knee et al. 2003, Mesaeli et al. 1999, Molinari et al. 2004, Nakamura et al. 2001) and conversely, calreticulin cannot compensate for the loss of calnexin (Denzel et al. 2002, Molinari et al. 2004, Zuppini et al. 2002). These observations indicate that calreticulin and calnexin are distinct proteins with very unique functions.

\section{CALRETICULIN AND CALNEXIN, ER LECTIN-LIKE CHAPERONES}

Calreticulin is a $\mathrm{Ca}^{2+}$-binding chaperone present in a number of extremely diverse species (Michalak et al. 1999). The protein binds/buffers $\mathrm{Ca}^{2+}$ in the ER lumen and participates in the folding of newly synthesized proteins and glycoproteins and it is an important constitute of a calreticulin/ calnexin cycle (Ellgaard et al. 1999, Jakob et al. 2001, Michalak et al. 1999, Saito et al. 1999). Figure 1 shows a model of calreticulin and calnexin. Calreticulin is made out of distinct structural and functional domains. The N-domain of calreticulin is globular with a disulfide bridge. This region binds heavy metals and may interact with a variety of molecules including proteins and nucleic acid. The P-domain comprises the proline-rich region, forms an extended arm structure and interacts with other ER luminal chaperones. The $\mathrm{C}$-domain is highly acidic, binds $\mathrm{Ca}^{2+}$ with high capacity and it is involved in $\mathrm{Ca}^{2+}$ storage in the lumen of the ER.

Several functions have been described for calreticulin, including regulation of $\mathrm{Ca}^{2+}$ homeostasis and $\mathrm{Ca}^{2+}$-dependent pathways (Arnaudeau et al. 2002, Camacho and Lechleiter 1995, Guo et al. 2002, Nakamura et al. 2001), lectin-like chaperone activity (Bergeron et al. 1994, Molinari and Helenius 2000, Nakamura et al. 2001), modulation of gene expression(Burns and Michalak 1993, Dedhar 1994, Michalak et al. 1999), nuclear transport (Holaska et al. 2001, Mesaeli and Phillipson 2004), and cell adhesion (Fadel et al. 1999, Fadel et al. 2001, Opas et al. 1996). It is generally believed that the protein has two principal functions: (i) molecular chaperone, and (ii) a modulator of $\mathrm{Ca}^{2+}$ homeostasis. Calreticulin functions as a molecular chaperone for 
many proteins and glycoproteins (Nauseef et al. 1995, Saito et al. 1999). The protein binds $\mathrm{Glc}_{1} \mathrm{Man}_{9} \mathrm{GlcNAc}_{2}$ oligosaccharide and recognizes the terminal glucose and four internal mannose in newly synthesized glycoproteins (Kapoor et al. 2003). Calreticulin binds $\mathrm{Ca}^{2+}$ and plays a role in regulation of $\mathrm{Ca}^{2+}$ homeostasis. Over-expression of calreticulin leads to increased amounts of $\mathrm{Ca}^{2+}$ in intracellular stores (Arnaudeau et al. 2002, Michalak et al. 1999, Nakamura et al. 2001), whereas calreticulin-deficient cells have reduced ER $\mathrm{Ca}^{2+}$ storage capacity (Nakamura et al. 2001). Store-operated $\mathrm{Ca}^{2+}$ influx across the plasma membrane is reduced in cells that express high level of calreticulin (Arnaudeau et al. 2002). Importantly, agonist-mediated $\mathrm{Ca}^{2+}$ release from the ER is inhibited in $\mathrm{crt}^{-/}$cells (Mesaeli et al. 1999, Nakamura et al. 2001). Problems with $\mathrm{Ca}^{2+}$ dependent signaling pathways must be responsible for embryonic (Mesaeli et al. 1999) and postnatal lethality seen in calreticulin-deficient mice (Guo et al. 2002).

Calnexin, is a type I integral membrane protein of the ER (Michalak et al. 2002, Wada et al. 1991). Calnexin can also be divided into distinct structural and functional domains (Fig. 1): N-terminal globular domain, extended arm P-domain, transmembrane domain and a short cytoplasmic tail (Wada et al. 1991). The N+P-domain of calnexin represents a "protein folding module" and it is attached to a membrane via a transmembrane domain. A major distinction between calreticulin and calnexin is that calnexin is an integral membrane protein (Fig. 1) whereas calreticulin is a luminal protein. Thus, calnexin interacts transiently with its protein-folding intermediates at the stationary phase of the ER membrane, whereas calreticulin interacts with its substrates in the mobile luminal environment. Different topological environments of calnexin and calreticulin must be critical in determining their distinct substrate specificities. calnexin has also been implicated to function as a modulator of ER $\mathrm{Ca}^{2+}$-ATPase (SERCA) in Xenopus oocytes (Roderick et al. 2000).

\section{CALRETICULIN/CALNEXIN CYCLE}

Calreticulin and calnexin, together with ERp57 constitute the calreticulin/calnexin cycle that is responsible for the folding and quality control of newly-synthesized glycoproteins (Fig. 2) (High et al. 2000, Zapun et al. 1999). There are other $\mathrm{Ca}^{2+}$ binding chaperones that reside in the lumen of the ER, including Grp94, the PDI family of proteins (PDI, ERp72), and BiP, but these proteins appear to play an important role in the folding and posttranslational modification of non-glycosylated proteins (Gething 1999, Nicchitta 1998). Calreticu-

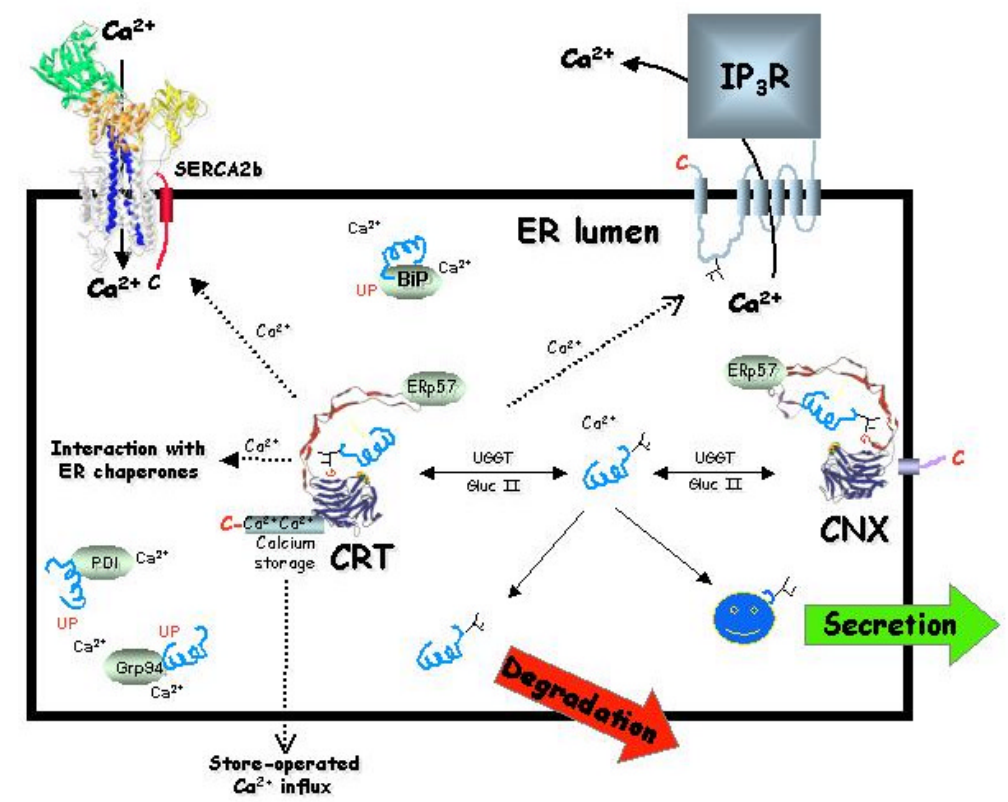

Fig. 2. Calreticulin/calnexin cycle.Calreticulin and calnexin bind monoglucosylated carbohydrate on newly-synthesized glycoproteins. Glucosidase II removes terminal glucose and UGGT recognizes misfolded glycoproteins and re-glucosylate them. This de-glucosylation-glucosylation cycle may be repeated several times before a newly synthesized glycoprotein is properly folded or sent for degradation. Calreticulin and calnexin also affect $\mathrm{Ca}^{2+}$ homeostasis. Calreticulin is a major $\mathrm{Ca}^{2+}$ storage protein and calnexin affects function of SERCA2b. Solid arrows indicate demonstrated pathways. Broken arrows indicate pathways not yet shown for mammalian cells. UP, unfolded glycoproteins, G, glucose residue. 
lin and calnexin are perhaps the most important chaperones because they directly affect the folding and posttranslational modification of virtually all glycosylated, secreted, or integral membrane proteins that pass through the ER.

Fig. 2 shows a diagram of calreticulin/calnexin cycle with emphasis on the role of calreticulin and calnexin in protein folding and modulation of $\mathrm{Ca}^{2+}$ homeostasis. Calreticulin and calnexin bind monoglucosylated carbohydrate on newlysynthesized glycoproteins (Trombetta 2003). The carbohydrate binding is sensitive to changes in $\mathrm{Ca}^{2+}$ concentration, suggesting that ER luminal $\mathrm{Ca}^{2+}$ may affect the folding of glycoproteins. ERp57 associates with the substrates recognized by both calreticulin and calnexin and catalyzes rearrangements of disulfide-bonds within the substrate proteins (Zapun et al. 1998). ERp57 docks onto the extended, P-domain of calreticulin and calnexin (Russell et al. 2004, Silvennoinen et al. 2004). Changes within the ER lumen, such as fluctuation in the concentration of $\mathrm{Ca}^{2+}, \mathrm{Zn}^{2+}$ or ATP, may affect the formation of these chaperone complexes and their ability to assist in protein folding (Corbett et al. 2000). For example, $\mathrm{Zn}^{2+}$ dependent conformational changes in calreticulin are known to enhance its carbohydrate-independent chaperone function (Saito et al. 1999).

\section{CALRETICULIN- AND CALNEXIN- DEFICIENT MICE}

Calreticulin deficiency is embryonic lethal due to a lesion in cardiac development (Guo et al. 2002, Mesaeli et al. 1999). Cells isolated from $\mathrm{crt}^{-/}$ embryos have impaired agonist-induced $\mathrm{Ca}^{2+}$ release (Nakamura et al. 2001), inhibited nuclear import of the transcription factor NF-ATc1, Mef2c and p53, modified sensitivity to apoptosis, compromised function of calnexin, and activated unfolded proteins response (UPR) (Li et al. 2002, Mesaeli et al. 1999, Mesaeli and Phillipson 2004, Nakamura et al. 2000, Nakamura et al. 2001) indicating a major impact of calreticulin deficiency on ER and cellular functions. Remarkably, $c r t^{-/}$ mice are rescued by expression of constitutively active calcineurin in the heart (Guo et al. 2002). Calcineurin is a $\mathrm{Ca}^{2+} /$ calmodulin-dependent serine/threonine phosphatase (Rusnak and Mertz 2000). It is a heterotetramer containing A and B subunits. Calcineurin-A is the catalytic subunit (Rusnak and Mertz 2000). A constitutively-active form of calcineurin (activated-calcineurin) can be generated by deletion of its C-terminal region (Rusnak and Mertz 2000). Cardiac expression of activated-calcineurin reversed the embryonic lethality seen in calreticulin-deficient mice and these mice exhibit severe postnatal pathology and die 7-35 days after birth (Guo et al. 2002). The rescue mice have severe growth retardation and metabolic problems (Guo et al. 2002). For example, they have elevated levels of both cholesterol and triacyglyceroles (TAG) (Guo et al. 2002). The underlying cause of the metabolic aberrations in these mice is not understood. $c r t^{-/}$mice serum lipids may be elevated because of compromised $\mathrm{Ca}^{2+}$ release from the ER and impaired function of ER associated molecules involved in lipid synthesis and uptake. Many metabolic processes rely of ER function therefore it is critical to understand a role of calreticulin and ER in postnatal metabolism and pathology.

In sharp contrast, calnexin deficiency is not embryonic lethal (Denzel et al. 2002). This is amazing considering great structural and functional similarities between the two chaperones. $c n x^{-/-}$ animals exhibit impaired motor function and die within the first 5 weeks of life (Denzel et al. 2002). $c n x^{-/}$mice are smaller than their siblings, develop sever motor problems of lower limb and difficulties with maintaining a proper balance.

Studies with calreticulin and calnexin gene knockout mice indicate that these proteins are unable to compensate for the loss of each other suggesting they must have unique functions (Denzel et al. 2002, Mesaeli et al. 1999, Nakamura et al. 2001). One function of calreticulin that cannot be compensated by calnexin is its role in modulation of $\mathrm{Ca}^{2+}$ homeostasis (Arnaudeau et al. 2002, Nakamura et al. 2001). We created viable crt $^{-}$ ${ }^{\prime}$ and $c n x^{-/}$cell lines indicating that in mammalian cell culture calreticulin and calnexin (and the calreticulin/calnexin cycle) are not essential for cell survival (Mesaeli et al. 1999, Scott and Dawson 1995). Deletion of glucosidase II in mammalian cells and glucosidase II and UGGT, key components of the calreticulin/calnexin cycle, in $S$. pombe has no serious consequences on cellular function (D'Alessio et al. 1999).

Yet, calnexin deficiency is lethal in $S$. pombe (Parlati et al. 1995a), S. cervisiae lacks most of the calnexin/calreticulin components (Parlati et al. 1995b). Calnexin and calreticulin deficiency is not lethal but it affects phagocytosis in Dictyostelium (Muller-Taubenberger et al. 2001) and promotes necrotic cell death in C. elegans (Xu et al. 2001). In summary, these findings support the hypothesis that calreticulin and calnexin are multifunctional proteins. Molecular chaperone function of calreticulin and calnexin may only partially explain phenotypes of $c n x^{-/-}$and $c r t^{-/}$mice. 


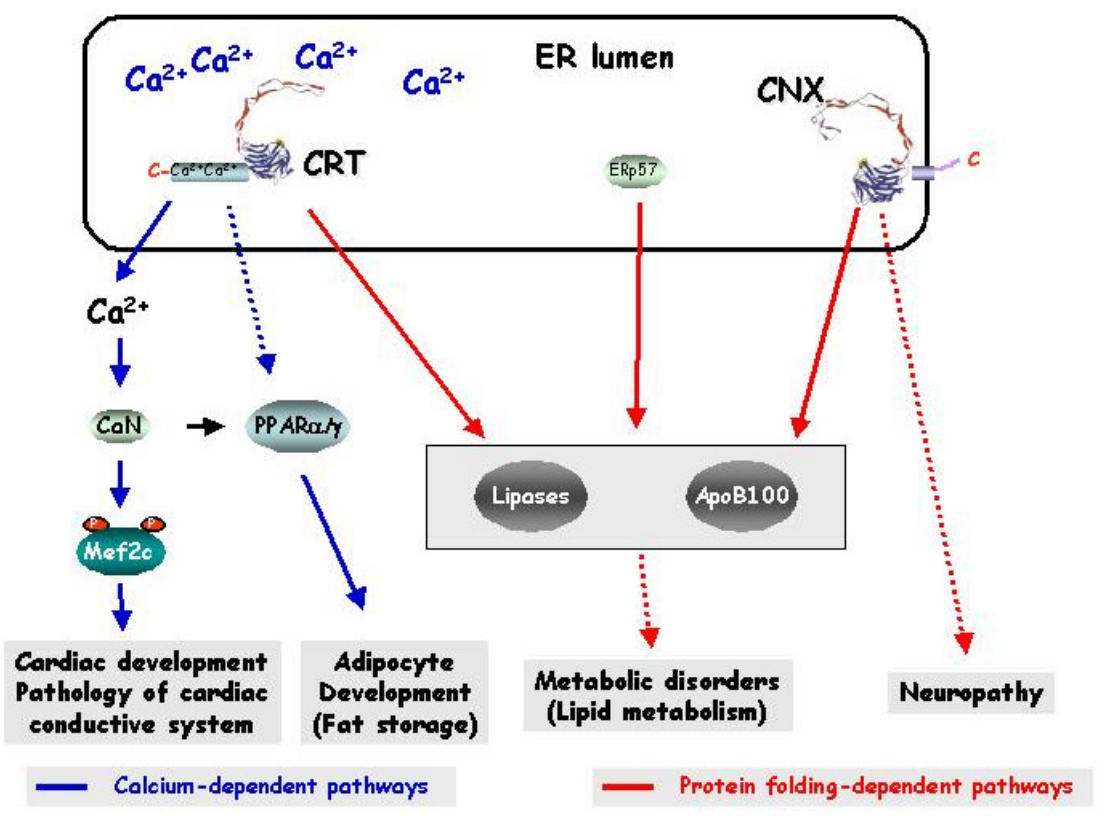

Fig. 3. A model of role of ER chaperones in development and metabolism. Schematic representation of relationship between calreticulin in the ER lumen, calnexin and developmental/metabolic pathways. Calreticulin affects many aspects of cardiac development and influences formation of adipose tissue. These effects are due to calreticulin function as regulator of $\mathrm{Ca}^{2+}$ homeostasis (blue). Calreticulin, ERp57 and calnexin also affects energy metabolism, especially lipid metabolism in mice. These effects of ER chaperones are likely due their role in protein folding (red).

\section{ER QUALITY CONTROL AND LIPID METABOLISM}

Analysis of chaperone-deficient mouse models revealed an important role of the ER quality control in regulation of energy metabolism. Calreticulin deficient mice die from a lesion in cardiac development. Animals rescued with activatedcalcineurin are born alive but exhibit serious metabolism problem (Guo et al. 2002). Furthermore, these animals have no significant adipose tissue indicting that calreticulin affect lipid metabolism and lipid storage after birth. In the absence of calreticulin there is a large increase in circulating lipids and lipoproteins (Guo et al. 2002). Preliminary experiments indicate that calreticulin's role in modulation of $\mathrm{Ca}^{2+}$ homeostasis affect adipogenesis but its chaperone function may modulate lipid synthesis and, ultimately fat metabolism (Fig. 3). In contrast low expression of ERp57 (heterozygote animals) or calnexin deficiency result in decrease levels of circulating lipoprotein particles and reduced level of serum cholesterol. These animals have also reduced level of ApoB100 suggesting that ERp57 and calnexin may be involved in folding and secretion of this lipoprotein. Chaperone function of ERp57 and calnexin may, therefore, play critical role in regulating lipid metabolism (Fig. 3). Future studies on the relationship between ER quality control and its chaperones should help us better understand the role of protein folding in the control and regulation of energy metabolism.

\section{ACKNOWLEDGEMENTS}

Research in our laboratory is supported by the Canadian Institutes of Health Research. The author is a Canadian Institutes of Health Research Senior Investigator.

\section{REFERENCES}

Arnaudeau S., Frieden M., Nakamura K. et al.: Calreticulin differentially modulates calcium uptake and release in the endoplasmic reticulum and mitochondria: J. Biol. Chem. 277:46696-46705, 2002.

Baumann O., Walz B.: Endoplasmic reticulum of animal cells and its organization into structural and functional domains. Int. Rev. Cytol. 205:149-214, 2001.

Bergeron J.J.M., Brenner M.B., Thomas D.Y., Williams D.B.: Calnexin: a membrane-bound 
chaperone of the endoplasmic reticulum. Trends Biochem. Sci. 19:124-128, 1994.

Burns, K., Michalak M.: Interactions of calreticulin with proteins of the endoplasmic and sarcoplasmic reticulum membranes. FEBS Lett. 318:181-185, 1993.

Camacho, P., Lechleiter J.D.: Calreticulin inhibits repetitive intracellular $\mathrm{Ca}^{2+}$ waves. Cell. 82:765-771, 1995.

Corbett E.F., Michalak M.: Calcium, a signaling molecule in the endoplasmic reticulum? Trends Biochem. Sci. 25:307-311, 2000.

Corbett E.F., Michalak K.M., Oikawa K. et al.: The conformation of calreticulin is influenced by the endoplasmic reticulum lumenal environment. J. Biol. Chem. 275:2717727185, 2000.

D'Alessio C., Fernandez F., Trombetta E.S., Parodi A.J.: Genetic evidence for the heterodimeric structure of glucosidase II. The effect of disrupting the subunit-encoding genes on glycoprotein folding. J. Biol. Chem. 274:25899-25905, 1999.

Dedhar S.: Novel functions for calreticulin: interaction with integrins and modulation of gene expression? Trends Biochem. Sci. 19:269-271, 1994.

Denzel A., Molinari M., Trigueros C., Martin J.E., Velmurgan S., Brown S., Stamp G., Owen M.J.: Early postnatal death and motor disorders in mice congenitally deficient in calnexin expression. Mol. Cell. Biol. 22:7398-7404, 2002.

Ellgaard L., Molinari M., Helenius A.: Setting the standards: quality control in the secretory pathway. Science 286:1882-1888, 1999.

Fadel M.P., Dziak E., Lo C.M. et al.: Calreticulin affects focal contact-dependent but not close contact-dependent cell-substratum adhesion. J. Biol. Chem. 274:15085-15094, 1999.

Fadel M.P., Szewczenko-Pawlikowski M., Leclerc P. et al.: Calreticulin affects ß-catenin associated pathways: J. Biol. Chem. 276:27083-27089, 2001.

Gething M.J.: Role and regulation of the ER chaperone BiP. Sem. Cell Dev. Biol. 10:465472, 1999.

Guo L., Nakamura K., Lynch J. et al.: Cardiacspecific expression of calcineurin reverses embryonic lethality in calreticulin-deficient mouse. J. Biol. Chem. 277:50776-50779, 2002.

High S., Lecomte F.J., Russell S.J. et al.: Glycoprotein folding in the endoplasmic reticulum: a tale of three chaperones? FEBS Lett. 476:38-41, 2000.

Holaska J.M., Black B.E., Love D.C. et al.: Calreticulin is a receptor for nuclear export. J. Cell Biol. 152:127-140, 2001.
Jakob C.A., Chevet E., Thomas D.Y., Bergeron J.J.: Lectins of the ER quality control machinery. Cell Differ. 33:1-17, 2001.

Kapoor M., Srinivas H., Kandiah E. et al.: Interactions of substrate with calreticulin, an endoplasmic reticulum chaperone. J. Biol. Chem. 278:6194-6200, 2003.

Knee R., Ahsan I., Mesaeli N. et al.: Compromised calnexin function in calreticulin deficient cells. Biochem. Biophys. Res. Commun. 304:661666, 2003.

Li, J., Puceat M., Perez-Terzic C.et al.: Calreticulin reveals a critical $\mathrm{Ca}^{2+}$ checkpoint in cardiac myofibrillogenesis. J. Cell Biol. 158:103-113, 2002.

Meldolesi J., Pozzan T.: The endoplasmic reticulum $\mathrm{Ca}^{2+}$ store: a view from the lumen. Trends Biochem. Sci. 23:10-14, 1998.

Mesaeli N., Phillipson C.: Impaired p53 expression, function, and nuclear localization in calreticulin-deficient cells. Mol. Biol. Cell 15: 1862-1870, 2004

Mesaeli N., Nakamura K., Zvaritch E. et al.: Calreticulin is essential for cardiac development. J. Cell Biol. 144:857-868, 1999.

Michalak M., Corbett E.F., Mesaeli N. et al.: Calreticulin: one protein, one gene, many functions. Biochem. J. 344:281-292, 1999.

Michalak M., Parker J.M.R., Opas M.: $\mathrm{Ca}^{2+}$ signaling and calcium binding chaperones of the endoplasmic reticulum. Cell Calcium. 32:269-278, 2002.

Molinari M., Helenius A.: Chaperone selection during glycoprotein translocation into the endoplasmic reticulum. Science 288:331-333, 2000.

Molinari M., Eriksson K.K., Calanca V. et al.: Contrasting functions of calreticulin and calnexin in glycoprotein folding and ER quality control. Mol. Cell. 13:125-135, 2004.

Muller-Taubenberger A., Lupas A.N. Li H. et al.: Calreticulin and calnexin in the endoplasmic reticulum are important for phagocytosis. EMBO J. 20:6772-6782, 2001.

Nakamura K., Bossy-Wetzel E., Burns K. et al.: Changes in endoplasmic reticulum luminal environment affect cell sensitivity to apoptosis. J. Cell Biol. 150:731-740, 2000.

Nakamura K., Zuppini A., Arnaudeau S. et al.: Functional specialization of calreticulin domains. J. Cell Biol. 154:961-972, 2001.

Nauseef W.M., McCormick S.J., Clark R.A.: Calreticulin functions as a molecular chaperone in the biosynthesis of myeloperoxidase. J. Biol. Chem. 270:47414747, 1995.

Nicchitta C.V.: Biochemical, cell biological and immunological issues surrounding the endoplasmic reticulum chaperone 
GRP94/gp96. Curr. Opin. Immunol. 10:103109, 1998.

Opas M., Szewczenko-Pawlikowski M., Jass G.K. et al.: Calreticulin modulates cell adhesiveness via regulation of vinculin expression. J. Cell Biol. 135:1913-1923, 1996.

Parlati F., Dignard D., Bergeron J.J.M., Thomas D.Y.: The calnexin homologue cnx1+ in Schizosaccharomyces pombe, is an essential gene which can be complemented by its soluble ER domain. EMBO J. 14:3064-3072, 1995a.

Parlati F., Dominguez M., Bergeron J.J.M., Thomas D.Y.: Saccharomyces cerevisiae CNE1 encodes an endoplasmic reticulum (ER) membrane protein with sequence similarity to calnexin and calreticulin and functions as a constituent of the ER quality control apparatus. J. Biol. Chem. 270:244-253, 1995 b.

Roderick H.L., Lechleiter J.D., Camacho P.: Cytosolic phosphorylation of calnexin controls intracellular $\mathrm{Ca}^{2+}$ oscillations via an interaction with SERCA2b. J. Cell Biol. 149:1235-1248, 2000.

Rusnak F., Mertz P.: Calcineurin: form and function. Physiol. Rev. 80:1483-1521, 2000.

Russell S.J., Ruddock L.W., Salo K.E.H., et al.: The primary substrate binding site in the $b^{\prime}$ domain of ERp57 is adapted for endoplasmic reticulum lectin association. J. Biol. Chem. 279:18861-18869, 2004.

Saito Y., Ihara Y., Leach M.R. et al.: Calreticulin functions in vitro as a molecular chaperone for both glycosylated and non-glycosylated proteins. EMBO J. 18:6718-6729, 1999.

Scott J.E., Dawson J.R.: MHC class I expression and transport in a calnexin-deficient cell line. J. Immunol. 155:143-148, 1995.
Silvennoinen L., Myllyharju J., Ruoppolo M. et al.: Identification and characterization of structural domains of human ERp57 - association with calreticulin requires several domains. J. Biol. Chem. 279:13607-1361, 2004.

Trombetta E.S.: The contribution of N-glycans and their processing in the endoplasmic reticulum to glycoprotein biosynthesis. Glycobiology. 13:77-91, 2003.

Trombetta E.S., Parodi A.J.: Quality control and protein folding in the secretory pathway. Annu. Rev. Cell Dev. Biol. 19:649-676, 2003.

Wada I., Rindress D., Cameron P.H. et al.: SSR alpha and associated calnexin are major calcium binding proteins of the endoplasmic reticulum membrane. J. Biol. Chem. 266:19599-19610, 1991.

Xu K., Tavernarakis N., Driscoll M.: Necrotic cell death in $C$. elegans requires the function of calreticulin and regulators of $\mathrm{Ca}^{2+}$ release from the endoplasmic reticulum. Neuron 31:957971, 2001.

Zapun A., Darby N.J., Tessier D.C. et al.: Enhanced catalysis of ribonuclease $\mathrm{B}$ folding by the interaction of calnexin or calreticulin with ERp57. J. Biol. Chem. 273:6009-6012, 1998.

Zapun A., Jakob C.A., Thomas D.Y., Bergeron J.J.M.: Protein folding in a specialized compartment: the endoplasmic reticulum. Structure Fold. Des. 7:R173-R182, 1999.

Zuppini A., Groenendyk J., Cormack L.A. et al.: Calnexin deficiency and endoplasmic reticulum stress-induced apoptosis: Biochemistry 41:2850-2858, 2002. 